\title{
The technique of utilizing a single gracilis functional muscle transfer to restore quadriceps function following sarcoma surgery
}

\author{
Christopher M. Nguyen ${ }^{1}$, Imran Ratanshi ${ }^{2}$, Jennifer L. Giuffre ${ }^{1}$, Edward W. Buchel', Thomas E. J. \\ Hayakawa $^{1}$
}

${ }^{1}$ Section of Plastic Surgery, Department of Surgery, University of Manitoba, Winnipeg R3A 1R9, Canada.

${ }^{2}$ The Plastic Surgery Group, Fraser Health Authority, Surrey, British Columbia V3V 1Z2, Canada.

Correspondence to: Dr. Thomas E. J. Hayakawa, FRCSC, Section of Plastic Surgery, Department of Surgery, University of Manitoba, 820 Sherbrook St,Winnipeg, Canada.E-mail: thplastic@hotmail.com

How to cite this article: Nguyen CM, Ratanshi I, Giuffre JL, Buchel EW, Hayakawa TEJ. The technique of utilizing a single gracilis functional muscle transfer to restore quadriceps function following sarcoma surgery. Plast Aesthet Res 2019;6:31.

http://dx.doi.org/10.20517/2347-9264.2019.42

Received: 24 Oct 2019 First Decision: 26 Nov 2019 Revised: 5 Dec 2019 Accepted: 12 Dec 2019 Published: 19 Dec 2019

Science Editor: Matthew L. lorio Copy Editor: Jing-Wen Zhang Production Editor: Jing Yu

\begin{abstract}
Aim: Quadriceps strength and knee extension, the most important factors limiting the ability to rise from a chair, are crucial for walking at an appropriate speed, ascending and descending stairs, and performing activities such as running, dancing, and jumping. Resection of the anterior compartment of the thigh, including all four quadriceps muscles, for the treatment of a sarcoma is uncommon; however, when necessary, it is very debilitating and adversely affects a patient's quality of life without functional reconstruction. Currently, there are a limited number of complex and difficult reconstructions to restore quadriceps function that have been described with variable outcomes. We describe a simple technique that employs a single gracilis functional muscle transfer to replace essential quadriceps function.
\end{abstract}

Methods: This is a case series describing the use of either a free or pedicled single gracilis muscle to restore quadriceps function following sarcoma resection.

Results: Four patients underwent an anterior compartment sarcoma resection that resulted in a large segmental defect and/or denervation of all four quadriceps muscles such that no quadriceps function would remain without reconstruction. All four patients underwent a functional reconstruction using a single gracilis. Three of the living patients achieved British Medical Research Counsel Grade 4 strength, can achieve full knee extension, are able to

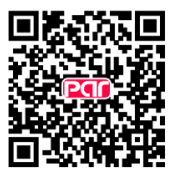


navigate stairs, and are able to ambulate without a brace. The fourth patient unfortunately was deceased in under three months following his tumor resection.

Conclusion: Despite its small size in comparison to the quadriceps muscles, with physiotherapy and training, the gracilis muscle demonstrates the capacity to hypertrophy and replace quadriceps function following limb salvage surgery.

Keywords: Knee extension, re-animation, functional muscle transfer, free flap, pedicled flap, sarcoma, microsurgery, quadriceps, reconstruction

\section{INTRODUCTION}

It is not uncommon to have to resect one or more components of the four quadriceps muscles during sarcoma resection from the anterior compartment of the thigh ${ }^{[1]}$. Pritsc et al. ${ }^{[2]}$ examined postoperative isometric strength and found that the strength of the quadriceps decreased by $22 \%, 33 \%, 55 \%$, and $76 \%$, respectively, when one, two, three, or more components of the quadriceps were resected ${ }^{[2,3]}$. Functional thresholds of quadriceps strength below which essential quadriceps functions are impaired have been difficult to determine as there are many other factors in addition to muscle strength that affect ambulatory ability ${ }^{[4]}$.

At our institution, in the setting of anterior compartment resections of the thigh for malignant soft tissue tumors, we do not routinely proceed with functional reconstruction or augmentation of the quadriceps muscles if at least one of the four quadriceps muscle groups remain intact. In those patients who undergo complete resection or complete loss of continuity (central wide resections) and/or denervation of all four quadriceps, we have been successful in restoring essential quadriceps function utilizing a single gracilis muscle transfer, either as a free flap from the contralateral leg, or, more recently, as a simple pedicled gracilis muscle from the ipsilateral leg. With either technique, the gracilis muscle is transferred into the rectus femoris position. With training, the gracilis is able to hypertrophy enough to perform essential quadriceps function.

\section{METHODS}

Institutional research ethics approval was obtained for the study (Ethics \#: HS23291). We retrospectively reviewed all patients at our institution who underwent complete resection and/or had complete denervation of all four quadriceps muscles as part of their sarcoma resection of the anterior thigh and reconstruction with a single gracilis muscle. All cases were performed by a single surgeon (Hayakawa TEJ). Patient demographics, surgical technique, and clinical outcomes such as British Medical Research Council (MRC) grading, knee extension, and ambulatory status is reported.

\section{Surgical technique}

The choice of free or pedicled gracilis is usually determined by the degree of cutaneous soft tissue reconstruction required. If there is a relatively small cutaneous defect, then both the functional quadriceps reconstruction and soft tissue reconstruction are accomplished by a single free contralateral gracilis myocutaneous flap. If the soft tissue or skin defect is too large for the gracilis skin paddle, then an ipsilateral pedicled gracilis is transferred into the rectus femoris position for the functional component of the reconstruction, and a larger cutaneous free tissue transfer such as an anterolateral thigh (ALT) or deep inferior epigastric perforator (DIEP) flap is added for coverage of the soft tissue defect.

In both scenarios, the inset of the gracilis is identical, and into the rectus femoris position. The gracilis will become the only "quadriceps" muscle and will provide both hip flexion and knee extension, which 

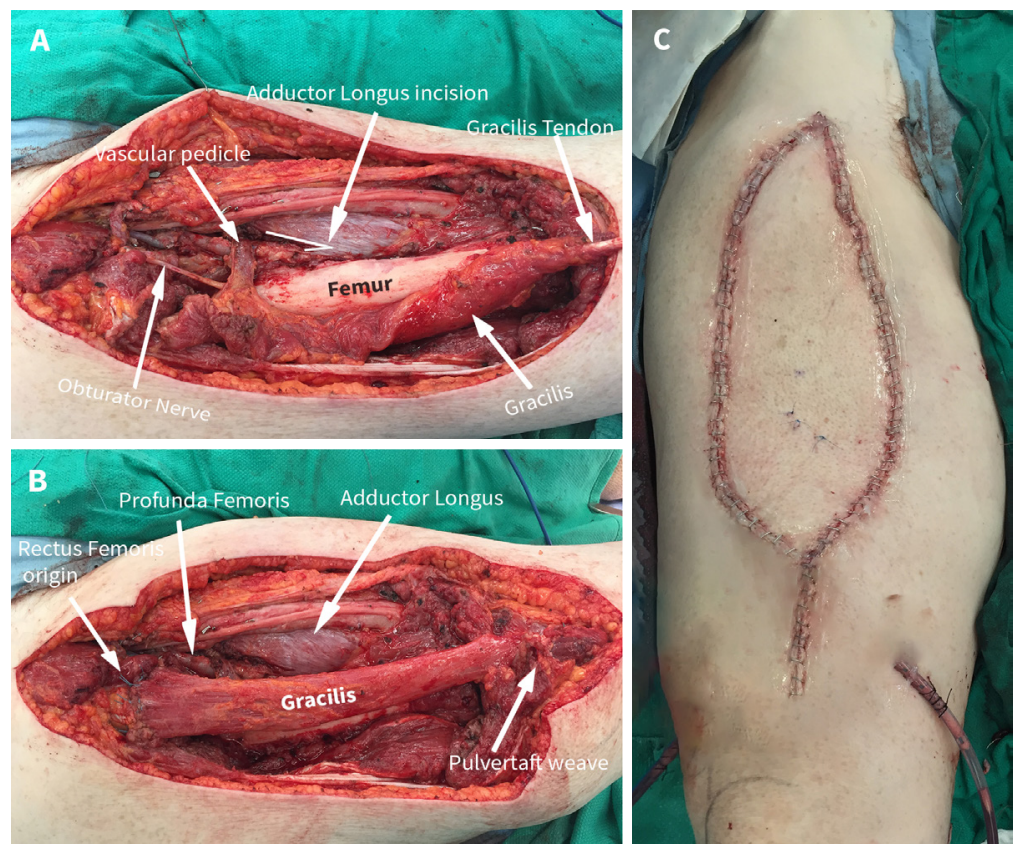

Figure 1. A: Pedicled gracilis harvest post-resection. Note the adductor longus releasing incision to prevent pedicle kinking following flap transposition allowing for tension-free inset; B: Gracilis inset in position of rectus femoris; C: Skin defect was covered with free anterolateral thigh flap

are both essential for normal gait. The entire length of the gracilis is harvested as previously described ${ }^{[5]}$ with care to harvest the proximal muscle directly off the periosteum of the ischium and the distal tendon off the pes anserine. There is almost always some of the rectus femoris tendon remaining proximally following the tumor resection. Occasionally additional rectus femoris muscle must be resected to get back to a solid tendinous portion that will allow several Krackow sutures as well as figure-of-eight sutures with o Ethibond ${ }^{\mathrm{TM}}$ (Ethicon Inc., Johnson \& Johnson, Somerville, NJ, USA) to secure the proximal gracilis muscle origin to the stout rectus femoris tendinous origin. The long distal gracilis tendon is then woven in a Pulvertaft fashion into the remaining quadriceps tendon complex distally.

When performing an ipsilateral pedicled gracilis, the vascular pedicle is dissected and freed to the profunda femoris artery and vein beneath the adductor longus. The "tunnel", deep to the adductor longus and superficial to the adductor brevis and magnus, may need to be elongated by making a "slit" or cut in the upper border of adductor longus that it is long enough to allow the gracilis muscle to be passed through from its normal position on the medial side of abductor longus to its new position on the lateral side. Care is taken to ensure there is no pressure or kinking of the gracilis vascular pedicle, which now must curve or arc up and over the profunda vessels as it follows the muscle during its transfer from the medial to the lateral side of the abductor longus. The gracilis is then placed into the rectus femoris position [Figures 1 and 2].

During flap harvest, we transect the motor branch of the obturator nerve to the gracilis and perform a neurorrhaphy to the most suitable available motor branch stump of the femoral nerve, which is ideally the remaining stump of the motor branch to the rectus femoris [Figure 3]. Of note, the sartorius muscle was resected in three cases and in no cases was it used for functional transfer. In no cases was nerve grafting to residual distal quadriceps elements performed.

The post-operative protocol consists of a five-day hospital stay to ensure flap viability and to educate patients in transfers in and out of a wheelchair. A Zimmer ${ }^{\mathrm{TM}}$ (Zimmer Biomet Holdings Inc. Warsaw, IN, USA) knee extension splint is fitted on the final hospital day with the knee in full extension. The patients are maintained in a wheelchair for two weeks post-operation to optimize wound healing and prevent seroma formation. At six weeks, the patients are switched from the Zimmer splint to an 

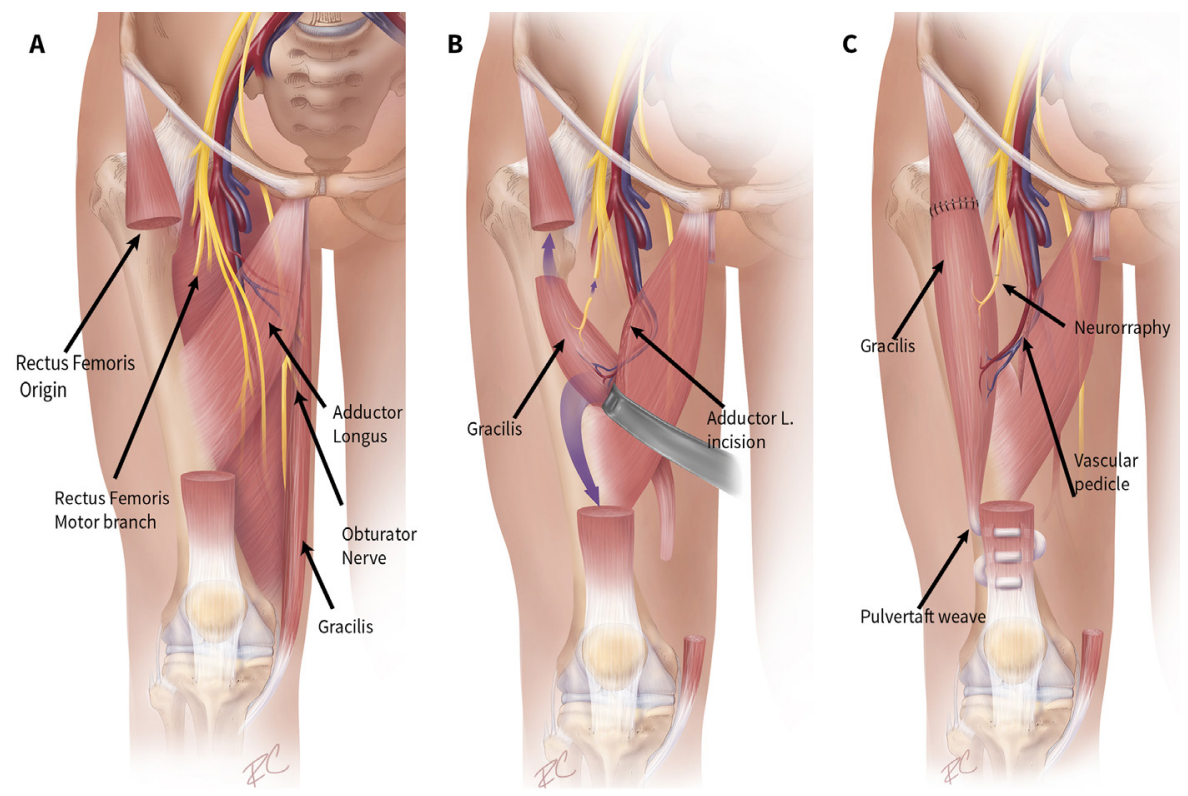

Figure 2. Artist rendition of surgical technique

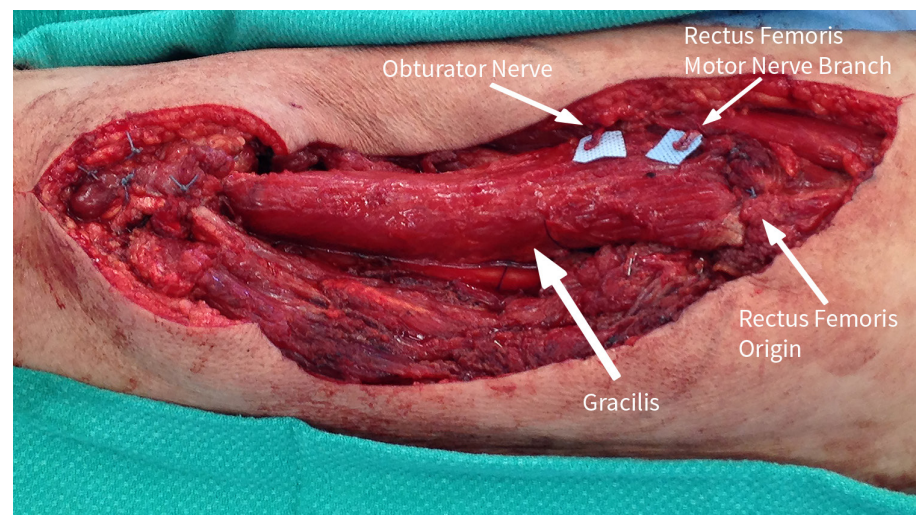

Figure 3. Neurorrhaphy of motor branch of obturator nerve to motor nerve of rectus femoris

adjustable hinged knee splint and begin range of motion exercises that increase by $20^{\circ}$ increments every week. Simultaneously motor frequency transcutaneous electrical nerve stimulation is begun. As soon as MRC grade 1 contraction is identified (a flicker of muscle contraction), the patients begin quadriceps strengthening exercises. Many standard strengthening techniques cannot be used because the knee is unstable from lack of quadriceps tone and therefore the ones shown in Figure 4 allow the patient to vary the degree of force on the new muscle as it gets stronger while also allowing them to provide stability to their knee by offloading forces onto their upper extremities.

\section{RESULTS}

We performed a gracilis muscle transfer to replace total quadriceps function in four patients with large central sarcoma resections resulting in large central gaps and/or denervation of all four quadriceps muscles. Patient's age at the time of surgery ranged from 24 to 65 years. Follow up in the three patients still alive ranged 3-20 years, with the single deceased patient surviving under three months following surgery and dying as a result of metastatic disease. All patients had high grade sarcomas, Fédération Nationale des Centres de Lutte Contre le Cancer Grade 3, and all surviving patients had negative margins 


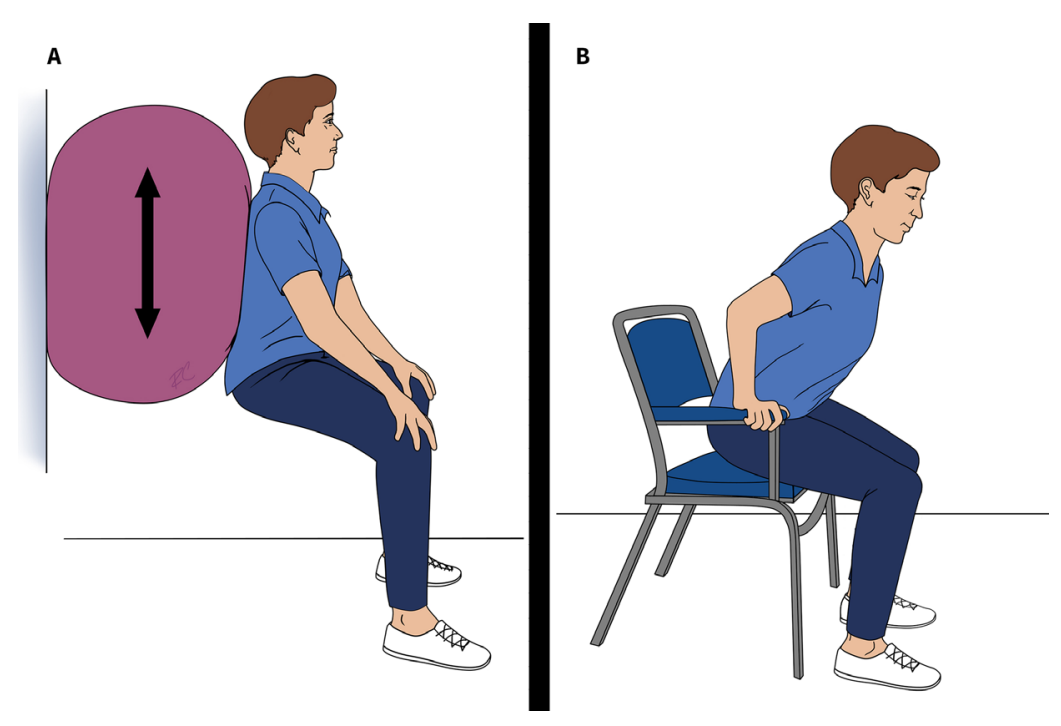

Figure 4. Quadriceps strengthening exercises post-reconstruction

at the time of initial resection. No patients had metastasis at the time of surgery. Two of the three patients continue to survive despite metastatic disease that occurred at three years following initial treatment in one, and eight years in the other. Both have pulmonary metastatic disease, and both have undergone treatment for their metastasis, one in the form of Video-Assisted Thoracoscopic Surgery and the other by Stereotactic Body Radiation Therapy. All patients underwent neoadjuvant radiation prior to surgery, and none of the surviving patients received chemotherapy. The reconstructions following resection included a free functional gracilis myocutaneous flap in one patient, a pedicled gracilis combined with an ALT flap in 2 patients, and a pedicled gracilis with a deep inferior epigastric artery perforator/superficial inferior epigastric artery (DIEP/SIEA) in one patient. The 3 surviving patients achieved MRC grade 4 muscle strength and were able to achieve full knee extension 12-18 months post-operation. The three surviving patients are able to ambulate without a brace and rise from the sitting position. All three of these patients demonstrated clinical signs of re-innervation with palpable muscle contraction beginning at, or just after 3 months post-operatively. Due to the clinical return of function, no electrophysiologic studies were performed. The exact length of the obturator nerve to the gracilis was not recorded, but in every case it was cut as short as possible to reduce re-innervation time yet still allow a tension free neurorrhaphy. One patient died from his disease less than three months post-operation, and therefore it was too early to demonstrate any contraction in the reinnervated functional reconstruction.

\section{Case examples}

The first case demonstrates a 50-year-old female with complete central resection of all four quadriceps [Figure 5]. At 18 months post-operation, this patient has enough strength from her gracilis to reach full extension, however she could not lock her knee into full extension for more than a short period of time until more strength and endurance was achieved after several additional months [Figure 6].

Her anticipated progress is demonstrated in the following videos. MRC grade 1 strength with contraction is noted at three months post-operation [Video 1]. MRC grade 2 contraction is noted at six months, which provides enough knee stability to discontinue the knee brace and allow the patient to ascend and descend stairs [Video 2]. MRC grade 3 strength is noted at nine months [Video 3]. MRC grade 4 strength is demonstrated at one year from surgery, which enables the patient to return to more vigorous sporting activities [Video 4]. Magnetic resonance imaging (MRI) demonstrates gracilis hypertrophy [Figure 7].

The second patient is a 22-year-old female with a large central resection of all four quadriceps muscles of left leg, reconstructed with a free gracilis muscle. Figure 8 demonstrates her "neo" quadriceps function with complete knee extension. She is able to complete a half marathon three years post-operation [Video 5]. 


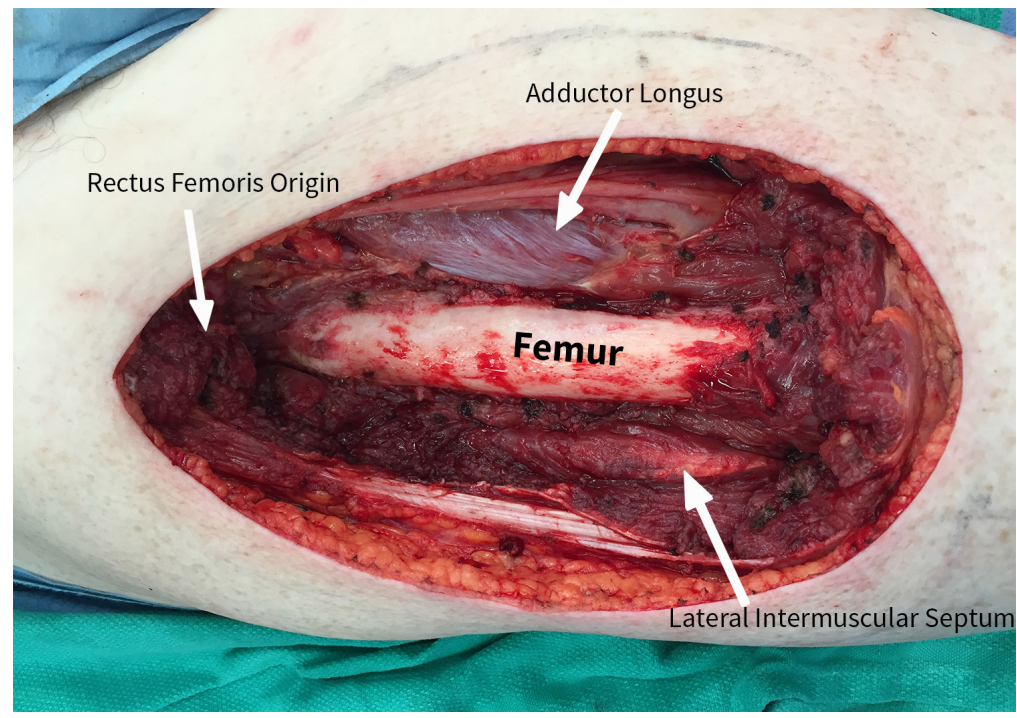

Figure 5. Post-oncologic resection of rectus femoris, vastus lateralis, vastus medialis, and vastus intermedius in a patient with a large high-grade sarcoma
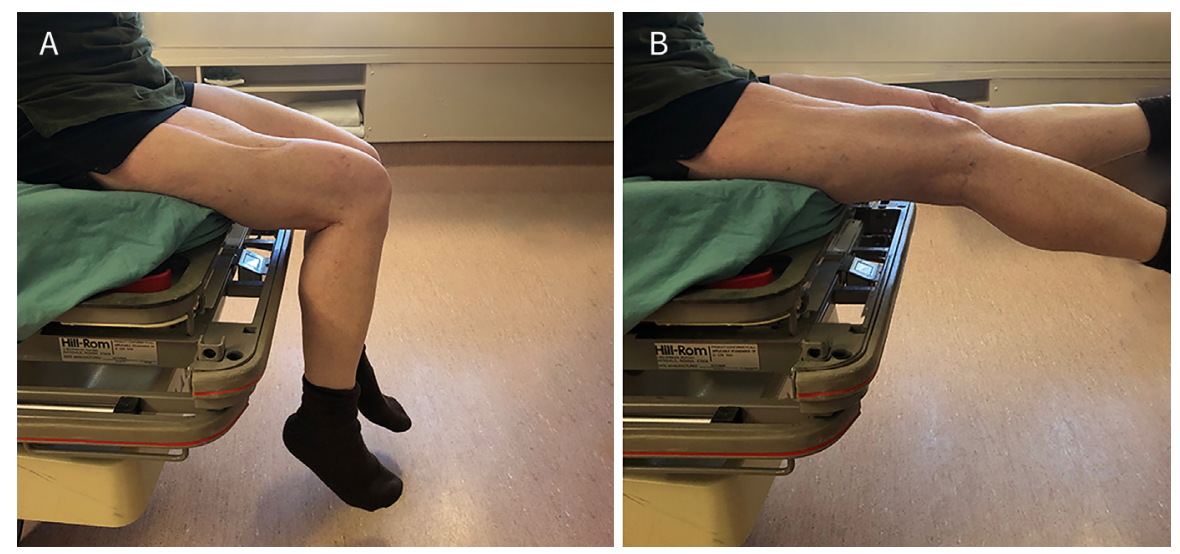

Figure 6. Grade 4 muscle strength at one year: extension of leg against gravity and resistance
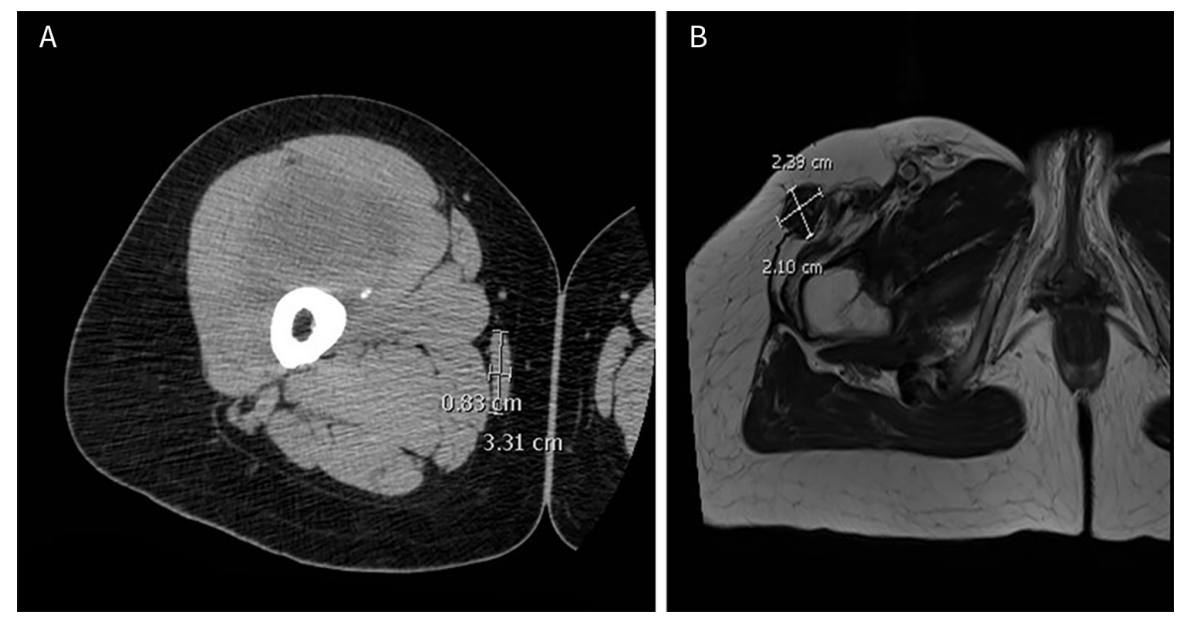

Figure 7. MRI showing right gracilis muscle hypertrophy post functional muscle transfer: (A) pre-operative MRI; and (B) 12-month postoperative MRI. MRI: magnetic resonance imaging 

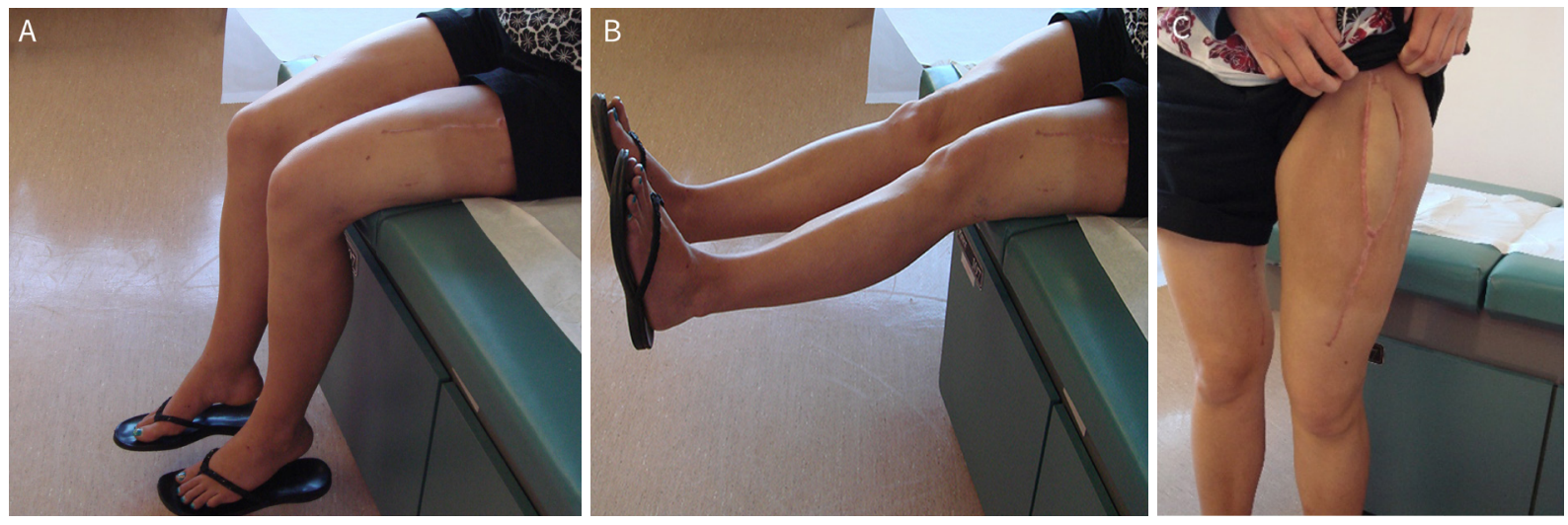

Figure 8. Demonstration of leg extension after free functional gracilis muscle flap to left quadriceps position at three years post-surgery: (A) leg at rest; (B) full active extension of leg; and (C) skin paddle of flap
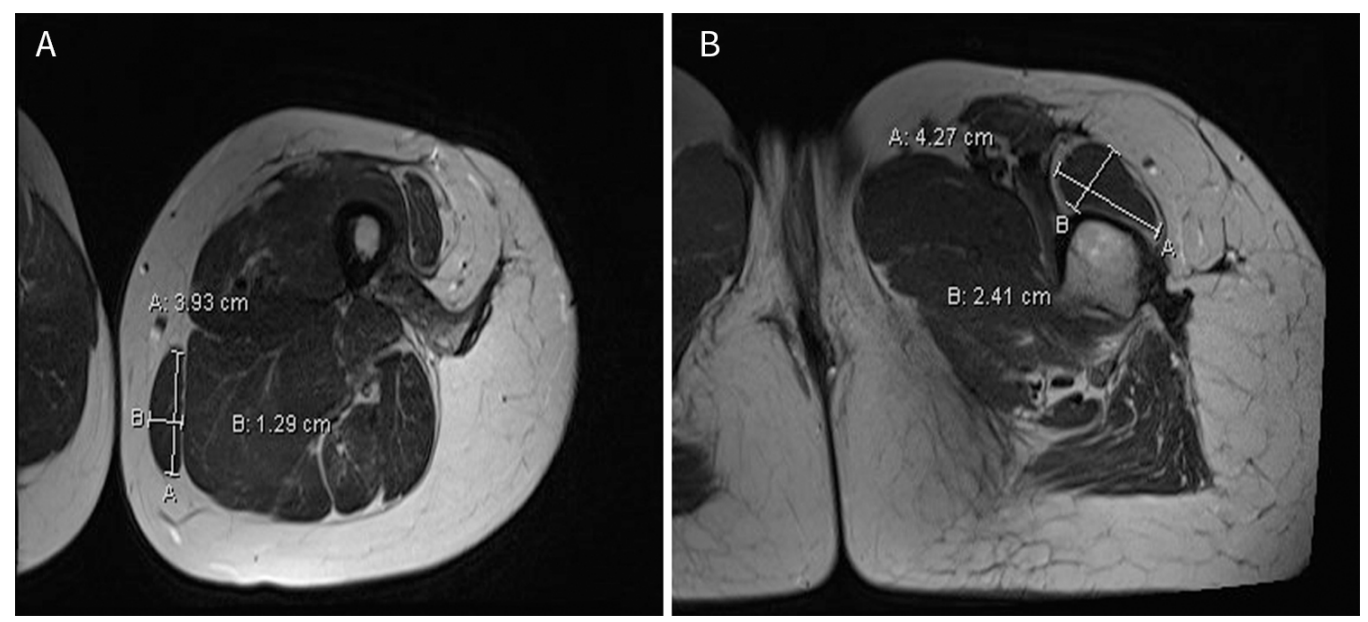

Figure 9. MRI showing gracilis muscle hypertrophy post free functional gracilis to left leg: (A) pre-operative MRI; and (B) 36-month postoperative MRI. MRI: magnetic resonance imaging

An MRI at three years post-surgery again shows that hypertrophy of the transferred gracilis muscle has occurred [Figure 9].

\section{DISCUSSION}

Although the anterior compartment of the thigh is the most common location for soft tissue sarcomas, complete resection of all four quadriceps muscles is uncommon ${ }^{[1,2]}$. Several different techniques have been described to reconstruct or augment remaining quadriceps function after tumor extirpation. Willcox et al. ${ }^{[6]}$ described good functional results following latissimus dorsi reconstruction in a 21-year-old following complete quadriceps resection and femoral prosthesis placement. Muramatsu et al. ${ }^{[7]}$, had only MRC grade 2 strength return following latissimus for a total quadriceps resection, but better results in those in which the latissimus was used to augment partial quadriceps defects. Innocenti et al. ${ }^{[8]}$ described four cases of complete quadriceps reconstruction with latissimus dorsi, but in all cases augmented the latissimus dorsi with the sartorius muscle and a large fascia lata patch to augment the muscle tendon repair. Pritsch et al. ${ }^{[2]}$ described combinations of biceps femoris, semitendinosis muscles, and sartorius to reconstruct partial quadriceps resections with good to excellent results in $86.7 \%$ of patients. Fischer et al.$^{[9]}$ also described local tendon transfers and utilized isolated biceps or a combination of biceps and semitendinosis or gracilis in 
43 patients. In this series, 9 of the 17 patients that were available for follow up evaluation had resection of greater than $3 / 4$ of the quadriceps or complete femoral nerve loss. All 43 patients in this series attained full knee extension; however, $41 \%$ still required walking aids. Only $14 \%$ of all patients underwent pre-operative neoadjuvant radiation therapy ${ }^{[9]}$. Despite its segmental innervation, Grinsell et al. described the use of the rectus abdominis muscle for complete quadriceps reconstruction. This group described dissecting 1-4 segmental intercostal nerves to the rectus abdominis over 10-12 cm and re-innervating the muscle to motor branches of the femoral nerve. They reported MRC Grade 4 power or greater in 6 of 11 patients ${ }^{[10]}$.

It has been our past practice not to proceed with functional reconstructions in those patients having at least one of the four quadriceps muscles remaining following tumor resection. Although these patients may be somewhat initially disabled, we noticed that with training and physiotherapy most are able to achieve unassisted ambulation, normal to near normal appearing gait, and full or nearly full knee joint extension. These findings are likely due at least in part to the well-established observation in both bodybuilding and powerlifting that muscles have the capacity for hypertrophy ${ }^{[11,12]}$. Based on these findings, we believed the gracilis would have the ability to replace enough quadriceps function essential for activities of daily living: sitting, getting up from a chair, and unassisted gait. To be clear, the goal of the transfer was not to replicate the strength and power of all four quadriceps muscles with a single smaller muscle.

From a surgical perspective, the gracilis is a simple, straight forward transfer, particularly if it is pedicled. The gracilis is in the same surgical field as the resection and hence there is no additional donor site morbidity, and no change of positioning or awkward positioning is required. The gracilis flap can be pedicled into the defect with no ischemia time and no microvascular anastomosis. The stout proximal fascia and long distal tendon make the gracilis perfectly suited for insertion into the rectus femoris origin proximally and into the quadriceps tendons distally with a strong Pulvertaft weave. The obturator nerve can be cut short for more rapid reinnervation if a long femoral nerve stump exists or tailored to be longer if the nerve was involved with the tumor more proximally. Thus far, we have always reinnervated the gracilis with the rectus femoris motor nerve branch of the femoral nerve, which is tagged during resection. Failure of reinnervation has not been a problem. Although not performed for sarcoma surgery, we have successfully performed pedicled functional gracilis leaving the obturator nerve to gracilis intact. We have utilized this in combination with a nerve transfer for complete femoral nerve injuries. In this case, the muscle dissection and placement are the same, but the obturator branch to the gracilis is left intact and the gracilis is "piggybacked" onto the medial side of rectus femoris. In this case, the obturator branch to the adductor longus is transected and used as a simultaneous nerve transfer to reinnervate the rectus femoris. Therefore, simply leaving the motor nerve to the gracilis intact during pedicled gracilis transfer may be another technique option but we do not have experience utilizing this in our sarcoma reconstructions.

There are advantages to the gracilis over the other free muscle transfers: its donor site functional deficit is likely less than that of a rectus abdominis, latissimus dorsi, or contralateral rectus femoris, and the recipient vessel location or the necessity of vein grafts in a radiated vessel depleted field does not become a factor when trying to accurately position the pedicled muscle transfer as compared to a free tissue transfer.

There are certainly situations in which other muscle transfers should be considered. For example, if the resection necessitates a femoral prosthesis, a latissimus dorsi or rectus abdominus is probably better suited to provide more complete coverage of the prosthesis. If the sartorius remains following resection, then it should also be considered in addition to the gracilis as described by Willcox et al. ${ }^{[6]}$ and Innocenti et al. ${ }^{[8]}$. Local tendon transfers can certainly be considered in institutions whose protocol does not include preoperative neoadjuvant radiation therapy. The risk of post-operative wound dehiscence and lymphedema with these techniques is not insignificant even in the non-irradiated setting ${ }^{[9]}$. 
We choose to place the gracilis in the rectus femoris position for several reasons. The ability to stand from a seated position is one of the most important measures of physical function and is essential for independent living ${ }^{[4,13]}$. The biarticulated rectus femoris is critically important in the sit-to-stand transition, as well as stepping and gait ${ }^{[1,15]}$. The rectus femoris is a two-joint muscle that acts both as a flexor of the hip and an extensor of the knee and is active during two phases of the gait cycle; in other words, it has bimodal activity ${ }^{[16-20]}$. The first burst of activity occurs during the loading response phase where it acts with the vasti by acting at the knee during load bearing to stabilize it ${ }^{[2]}$. The second burst occurs during the pre- and initial-swing phase of the gait cycle where it acts as a hip flexor in propelling the limb forward into swing ${ }^{[21]}$. These unconscious complex sets of coordinated movements are the reason we chose to reinnervate the gracilis with the motor nerve to rectus femoris rather than simply transfer it with its obturator innervation intact and hope that retraining could occur. The three vasti muscles are important for standing function and they extend the knee without flexing the thigh ${ }^{[22]}$. Likely because of this, two of our functional gracilis patients did have symptoms of fatigue if standing for longer periods of time. The vastus lateralis and intermedius are the strongest vasti; however, the vastus medialis is important for locking the knee in terminal extension and preventing patellar drift and lateral subluxation ${ }^{[22]}$. Considering this, our patients could all reach complete knee extension while seated on a bench but only one could hold their knee in full extension for more than a brief period of time. Interestingly, we did not have any problems with patellar subluxation or drift, and this may be attributed to the long distance distally that we weaved the gracilis tendon into the quadriceps tendon complex as well as some stabilizing fibrosis from the neo adjuvant radiotherapy that may have occurred.

In summary, the required amount of quadriceps strength necessary to maintain quality of life has not been accurately established ${ }^{[4]}$. It remains unclear which muscle or muscle transfers in the body are suitable to replace enough quadriceps strength and function to achieve this endpoint. Although the gracilis muscle is clearly not as strong as the quadriceps muscle complex, there are other clinical examples where a much smaller and weaker muscle can replace the essential functions of a much larger muscle group. An example would be the scenario of total biceps and brachialis resection or denervation in which a much smaller and weaker brachioradialis can adequately compensate for elbow flexion such that no additional reconstruction is usually required. Our experience suggests that with physiotherapy and training, and in the appropriate patient, the gracilis has enough capacity to provide essential quadriceps function following complete resection and/or denervation associated with limb salvage sarcoma surgery.

\section{DECLARATIONS}

\section{Authors' contributions}

Concept study design, literature search, and manuscript writing: Hayakawa TEJ

Manuscript preparation, data acquisition: Nguyen CM, Ratanshi I

Manuscript review: Giuffre JL, Buchel EW

\section{Availability of data and materials}

IRB approved retrospective study based on University of Manitoba Health Sciences Centre hospital charted data.

Financial support and sponsorship

None.

\section{Conflicts of interest}

All authors declared that there are no conflicts of interest. 


\section{Ethical approval and consent to participate}

IRB approval prior to data acquisition and study design (HS23291).

\section{Consent for publication}

A written informed consent for publication of videos and photos were obtained.

\section{Copyright}

(c) The Author(s) 2019.

\section{REFERENCES}

1. Enneking WF, Spanier SS, Malawer MM. The effect of the anatomic setting on the results of surgical procedures for soft parts sarcoma of the thigh. Cancer 1981;47:1005-22.

2. Pritsch T, Malawer MM, Wu CC, Squires MH, Bickels J. Functional reconstruction of the extensor mechanism following massive tumor resections from the anterior compartment of the thigh. Plastic Reconstr Surg 2007;120:960-9.

3. Markhede G, Stener B. Function after removal of various hip and thigh muscles for extirpation of tumors. Acta Orthop Scand 1981;52:373-95.

4. Ploutz-Snyder LL, Manini T, Ploutz-Snyder RJ, Wolf DA. Functionally relevant thresholds of quadriceps femoris strength. J Gerontol A Biol Sci Med Sci 2002;57:B144-52.

5. Giuffre JL, Bishop AT, Shin AY. Harvest of an entire gracilis muscle and tendon for use in functional muscle transfer: a novel technique. J Reconstr Microsurg 2012;28:349-58.

6. Willcox TM, Smith AA, Beauchamp C, Meland NB. Functional free latissimus dorsi muscle flap to the proximal lower extremity. Clin Orthop Relat Res 2003:285-8.

7. Muramatsu K, Ihara K, Miyoshi T, Yoshida K, Hashimoto T, Taguchi T. Transfer of latissimus dorsi muscle for the functional reconstruction of quadriceps femoris muscle following oncological resection of sarcoma in the thigh. JPRAS 2011;64:1068-74.

8. Innocenti M, Abed YY, Beltrami G, Delcroix L, Balatri A, et al. Quadriceps muscle reconstruction with free functioning latissimus dorsi muscle flap after oncological resection. Microsurgery 2009;29:189-98.

9. Fischer S, Soimaru S, Hirsch T, Kueckelhaus M, Seitz C, et al. Local tendon transfer for knee extensor mechanism reconstruction after soft tissue sarcoma resection. JPRAS 2015;68:729-35.

10. Grinsell D, Lonie S, Wilson KC, Choong PFM. The innervated rectus abdominis flap for quadriceps reconstruction. JPRAS 2019;72:941-5.

11. Schoenfeld BJ. The mechanisms of muscle hypertrophy and their application to resistance training. J Strength Cond Res 2010;24:2857-72.

12. Schoenfeld BJ. Potential mechanisms for a role of metabolic stress in hypertrophic adaptations to resistance training. Sports Med 2013;43:179-94.

13. Riley PO, Schenkman ML, Mann RW, Hodge WA. Mechanics of a constrained chair-rise. J Biomech 1991;24:77-85.

14. Kobetic R, Triolo RJ, Uhlir JP, Bieri C, Wibowo M, et al. Implanted functional electrical stimulation system for mobility in paraplegia: a follow-up case report. IEEE Trans Rehabil Eng 1999;7:390-8.

15. Sharma M, Marsolais EB, Polando G, Triolo RJ, Davis JA Jr, et al. Implantation of a 16-channel functional electrical stimulation walking system. Clin Orthop Relat Res 1998:236-42.

16. Perry J, Davids JR. Gait analysis: normal and pathological function. J Pediatr Orthop 1992;12:815.

17. Murray MP, Mollinger LA, Gardner GM, Sepic SB. Kinematic and EMG patterns during slow, free, and fast walking. J Orthop Res 1984;2:272-80.

18. Shiavi R, Bugle HJ, Limbird T. Electromyographic gait assessment, Part 1: Adult EMG profiles and walking speed. J Rehabil Res Dev 1987;24:13-23.

19. Ericson MO, Nisell R, Ekholm J. Quantified electromyography of lower-limb muscles during level walking. Scand J Rehabil Med 1986;18:159-63.

20. Csongradi J, Bleck E, Ford WF. Gait electromyography in normal and spastic children, with special reference to quadriceps femoris and hamstring muscles. Dev Med Child Neurol 1979;21:738-48.

21. Annaswamy TM, Giddings CJ, Della Croce U, Kerrigan DC. Rectus femoris: its role in normal gait. Arch Phys Med Rehabil 1999;80:930-4.

22. Gustafson KJ, Pinault GCJ, Neville JJ, Syed I, Davis JA Jr, et al. Fascicular anatomy of human femoral nerve: implications for neural prostheses using nerve cuff electrodes. J Rehabil Res Dev 2009;46:973-84. 